\title{
Effects of $\alpha$-Methyl-Para-Tyrosine (AMPT) in Drug-Free Depressed Patients
}

\author{
Helen L. Miller, M.D., Pedro L. Delgado, M.D., Ronald M. Salomon, M.D., \\ George R. Heninger, M.D., and Dennis S. Charney, M.D.
}

A variety of biologic studies have demonstrated abnormal regulation of the norepinephrine (NE) system in patients with major depression, suggesting a role for NE in the etiology of depression. Brain NE and dopamine levels can be rapidly reduced by blocking synthesis with the tyrosine hydroxylase inhibitor $\alpha$-methyl-para-tyrosine (AMPT). In the current investigation, AMPT was administered to drug-free depressed patients to evaluate the effect on mood of diminished catecholamine levels.

Seventeen drug-free patients meeting DSM-III-R criteria for major depressive episode were tested with AMPT and an active placebo control, diphenhydramine. Testing was accomplished in a double-blind, crossover fashion, with random assignment to test conditions. Each test included baseline evaluation, 2 days with administration of either AMPT or diphenhydramine, and a follow-up day. Diphenhydramine was used as an active control because of the significant sedation associated with AMPT. Behavioral ratings, including visual analogue scales for a variety of feeling states, the Hamilton Depression Rating Scale (HDRS), and plasma for 3-methoxy-4-hydroxyphenelethyleneglycol (MPHG) and homovanillic acid (HVA) levels, were obtained.

AMPT significantly reduced plasma HVA by $70 \%$ and MHPG by $50 \%$, but it had no significant effects on the HDRS. AMPT also significantly increased visual analogue ratings of "tired" and decreased ratings of "energetic." Diphenhydramine significantly decreased HDRS scores, but the change was small and was not clinically apparent.

The lack of AMPT effects on depressed mood, in conjunction with a prior report that large reductions in plasma tryptophan do not systematically alter depressed mood, indicate that monoamine deficiency by itself is insufficient explanation of the cause of depression. The role of the noradrenergic system needs to be considered in relationship to the many other neurobiologic factors that could be involved in the pathophysiology of depression. [Neuropsychopharmacology 14:151-157, 1996]
KEY WORDS: AMPT; Norepinephrine; Dopamine; Depressive disorder

From the Department of Psychiatry, Yale University School of Medicine, New Haven, Connecticut; West Haven Department of Veterans Affairs Medical Center, West Haven, Connecticut (HLM, PLD, RMS, GRH, DSC); Department of Veterans Affairs Medical Center, Psychiatry Service 116A1, West Haven VA Medical Center, West Haven, CT (HLM, PLD, RMS, DSC); and Connecticut Mental Health Center, New Haven, CT (GRH).

Presented in part at the Annual Meeting of the American Psychiatric Association, May 1993, San Francisco, California and at the Annual Meeting of the American College of Neuropsychopharmacology, December 1993, Honolulu, Hawaii.

Address correspondence to: Helen L. Miller, M.D., Affective Disorder Program, Veterans Affairs Medical Center-116A, 950 Campbell Avenue, West Haven, CT 06516.

Received October 11, 1994; revised March 31, 1995; accepted April 26, 1995.
Over the last 3 decades, the catecholamine hypothesis of the pathophysiology of depression has taken on several forms. Initially, the hypothesis postulated that a deficiency of norepinephrine (NE) was responsible for depression. This form of the hypothesis was based on clinical observations of the mood-lowering effects of drugs such as reserpine that deplete catecholamines, as well as the clinical efficacy of the monoamine oxidase inhibitors and tricyclic antidepressants, which increase the synaptic availability of catecholamines (Bunney and Davis 1966; Schildkraut 1965).

Subsequently, as a result of preclinical investigations of antidepressant mechanisms of action, the hypothesis was refined to include alterations in noradrenergic receptor function. It has been suggested that 
enhanced sensitivity of presynaptic $\alpha_{2}$-adrenergic (Garcia-Sevilla et al. 1966) and postsynaptic $\beta$-adrenergic receptors (Mann et al. 1986), and reduced sensitivity of postsynaptic $\alpha_{2}$-adrenergic receptors (Siever 1987) may be related to the pathophysiology of depression. It has also been suggested that depression results from a dysregulation of the noradrenergic neuronal system and that antidepressants act to return the systems to a state of equilibrium (Siever and Davis 1985).

A comprehensive synthesis of noradrenergic function in affective disorders is beyond the scope of this report; appropriate references should be consulted for further information on this topic (Delgado et al. 1992; Siever 1987). Clinical evidence has not consistently supported the hypotheses of specific abnormalities in presynaptic regulation of noradrenergic system activity or postsynaptic $\beta$-adrenergic receptor function. A blunted growth hormone response to the $\alpha_{2}$-adrenergic receptor agonist clonidine has been found in depression, panic disorder, and substance abuse, suggesting that abnormalities in postsynaptic noradrenergic receptor function are not specific to affective illness (Delgado et al. 1992; Siever 1987).

There are also data suggesting that dopamine (DA) may be an important neurotransmitter in the regulation of mood. Preclinical studies have shown that brain reward systems involve DA. Clinical investigations have reported that cerebrospinal fluid levels of homovanillic acid (HVA), a major metabolite of DA are reduced in depressed patients. Patients with Parkinson's disease have a high frequency of associated depression (Kapur and Mann 1992). Moreover, nomifensine, an antidepressant that inhibited dopamine reuptake in addition to its other pharmacologic properties, was an effective antidepressant.

The purpose of the present investigation was to determine the behavioral effects of $\alpha$-methyl-paratyrosine (AMPT) in depressed patients. AMPT decreases NE and DA levels via inhibition of tyrosine hydroxylase, a rate-limiting enzyme in the synthesis of both norepinephrine and dopamine (Widerlov and Lewander 1978). Thus, the administration of AMPT to depressed patients would allow an evaluation of the effects of NE and DA depletion on the severity of depressed mood and other associated symptoms of depressive disorders. Our a priori hypothesis was that AMPT would produce an exacerbation of depression.

\section{METHODS}

\section{Experimental Subjects}

Eighteen drug-free patients, meeting Diagnostic Statistical Manual III-R (DMS-III-R) criteria for major depressive episode (American Psychiatric Association 1987), were studied after having given written informed con- sent. Four women, mean age 30 , and 13 men, mean age 42 , were included in the study. Patients were either inpatients $(N=2)$ on the Affective Disorders Unit or outpatients $(N=16)$ in the Mood Disorders Clinic of the West Haven VA Medical Center. Depressed patients were selected only if they satisfied DSM-III-R criteria for current major depressive episode, and had a minimum score of at least 20 on the 25-item, modified Hamilton Depression Rating Scale (HDRS) (Mazure et al. 1986). Patients with current or past history of psychotic symptoms were not studied.

Diagnoses were established by consensus between two investigators. Severity of depression was assessed with the HDRS, using a structured interview, the Yale Clinical Depression Inventory (Mazure et al. 1986). All patients were in good general medical health and had not abused substances for 6 months prior to testing. Demographic and diagnostic data are presented in Table 1.

\section{AMPT Testing}

Subjects received AMPT and diphenhydramine control testing 1 week apart. Assignment to test conditions was random and double-blind. One-half of subjects had AMPT testing first; the other half had diphenhydramine control testing first. Because of significant sedation associated with AMPT testing, diphenhydramine was used as an active control rather than an inactive placebo.

Each test sequence consisted of 4 days: a baseline test day during which no medication was given (day 1), two days of treatment with either AMPT $1 \mathrm{~g}$ t.i.d. (day 2), and AMPT $1 \mathrm{~g}$ b.i.d. (last dose given at 12:00 P.M.; day 3), or diphenhydramine $50 \mathrm{mg}$ t.i.d. (day 2) and $50 \mathrm{mg}$ b.i.d. (day 3), and a post-test evaluation (day 4). Subjects were given a low monoamine diet for the 4 days of testing in order to minimize variability in catecholamine turnover. They had serum collected each morning (9:00 A.M.) for determination of MHPG and HVA levels. Behavioral ratings were obtained once on day 1 (9:00 A.M.), twice a day during medication administration (9:00 A.M. and 4:30 P.M., days 2 and 3), and once on the follow-up day (9:00 A.M., day 4).

\section{Behavioral Ratings}

Depressive symptoms were assessed with the modified 25-item HDRS, using a structured interview, the Yale Depression Inventory (Mazure et al. 1986). Clinicianand patient-rated analogue scales (100-mm lines) were obtained for 12 mood states (talkative, happy, drowsy, nervous, sad, calm, depressed, anxious, energetic, fearful, mellow, high, angry, irritable, tired, and hungry). 
Table 1. Demographic Characteristics of Patients

\begin{tabular}{|c|c|c|c|c|c|c|}
\hline Patient & Sex & Age & $\begin{array}{l}\text { Current } \\
\text { Axis I Diagnosis }\end{array}$ & $\begin{array}{c}\text { Lifetime } \\
\text { Substance Abuse }^{a}\end{array}$ & $\begin{array}{c}\text { Prior } \\
\text { Hospitalizations }\end{array}$ & $\begin{array}{c}\text { Baseline Score } \\
\text { Hamilton } \\
\text { Depression } \\
\text { Rating Scale }\end{array}$ \\
\hline 1 & $\mathrm{~F}$ & 21 & Major depression & None & None & 26 \\
\hline 2 & $\mathrm{~F}$ & 25 & Major depression & Cocaine abuse & None & 43 \\
\hline 3 & $\mathrm{~F}$ & 45 & $\begin{array}{l}\text { Major depression, } \\
\text { dysthymia, } \\
\text { simple phobia }\end{array}$ & Alcohol abuse & None & 32 \\
\hline 4 & $\mathrm{~F}$ & 27 & $\begin{array}{l}\text { Major depression, } \\
\text { anorexia, bulimia }\end{array}$ & None & None & 35 \\
\hline 5 & M & 23 & Major depression & Alcohol abuse & None & 22 \\
\hline 6 & M & 34 & Bipolar, depressed & Cocaine, alcohol abuse & 3 & 43 \\
\hline 7 & M & 35 & Bipolar, depressed & Polysubstance abuse & 3 & 22 \\
\hline 8 & M & 35 & $\begin{array}{l}\text { Major depression, } \\
\text { dysthymia }\end{array}$ & Alcohol abuse & None & 32 \\
\hline 9 & M & 38 & Major depression & None & 2 & 48 \\
\hline 10 & M & 41 & Major depression & None & None & 26 \\
\hline 11 & M & 42 & $\begin{array}{l}\text { Major depression, } \\
\text { generalized } \\
\text { anxiety disorder }\end{array}$ & $\begin{array}{l}\text { Polysubstance abuse } \\
\text { (alcohol, LSD, } \\
\text { cocaine, marijuana) } \\
\text { in remission } \times 6 \text { yrs }\end{array}$ & $\begin{array}{l}3 \text { (for poly } \\
\quad \text { substance abuse) }\end{array}$ & 33 \\
\hline 12 & $\mathrm{M}$ & 43 & $\begin{array}{l}\text { Major depression, } \\
\text { PTSD, simple } \\
\text { phobia panic } \\
\text { disorder }\end{array}$ & None & None & 32 \\
\hline 13 & $\mathrm{M}$ & 43 & $\begin{array}{l}\text { Major depression, } \\
\text { PTSD }\end{array}$ & None & None & 41 \\
\hline 14 & $\mathrm{M}$ & 45 & Major depression & None & None & 23 \\
\hline 15 & M & 48 & Major depression & Alcohol abuse & None & 23 \\
\hline 16 & $\mathrm{M}$ & 58 & Major depression & None & None & 30 \\
\hline 17 & M & 63 & Major depression & $\begin{array}{l}\text { Alcohol } 20 \text { yrs } \\
\text { prior to admission }\end{array}$ & 1 & 32 \\
\hline
\end{tabular}

${ }^{a}$ None of the patients had abused substances for six months prior to study participation.

\section{Biochemical Assays}

Plasma-free levels of HVA (interassay $\mathrm{CV}=8 \%$, intraassay $\mathrm{CF}=4 \%$ ) (Bacopoulos et al. 1979) and 3-methoxy-4hydroxyphenelethyleneglycol (MHPG) (interassay CV = $11 \%$, intraassay $C F=6 \%$ ) (Maas et al. 1976) were measured by gas chromatography and mass spectrometry, with the use of deuterated internal standards.

\section{Dropouts}

One subject experienced a severe panic attack approximately 30 hours after the last dose of AMPT. His symp- toms included extreme anxiety, depression, and motoric restlessness. This subject did not complete the control testing and is not included in the data analysis. Seventeen subjects completed both AMPT and diphenhydramine control testing.

\section{Data Analysis}

The effects of AMPT on behavior, and on MHPG and HVA levels, were initially analyzed using analysis of variance for repeated measures. This allowed an assessment of the main effects of drug (AMPT or diphenhydramine), time (changes over time points sampled), and 


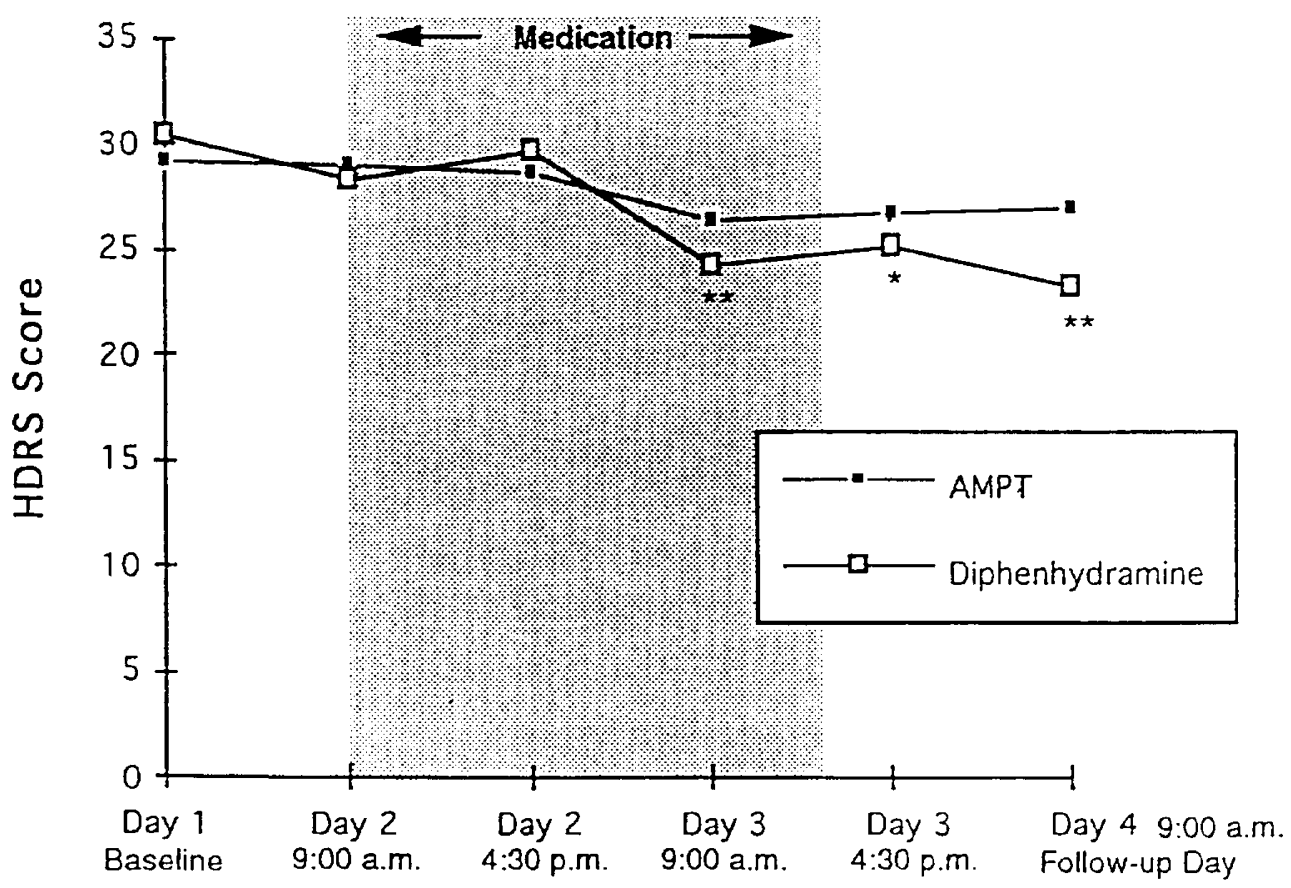

AMPT or Diphenhydramine Administration

Figure 1. The effect of AMPT and diphenhydramine on HDRS scores in depressed patients. There was a significant drug and time interaction $(F=3.74, d f=3, p<.017)$. There was a significant $(p<.05)$ decrease in the HDRS from baseline after diphenhydramine, as indicated by one asterisk. Time points marked with two asterisks were significant at $p<.01$. There was no significant change from baseline in the HDRS during AMPT administration.

the drug and time interaction. Post hoc analyses to examine changes at specific time points were calculated using the Dunnett's multiple $t$ tests. Results are reported as significant when $p<.05$ with a two-tailed test. Data analysis and graphic representations utilized the Statistical Program for the Social Sciences ${ }^{\oplus}$ (SPSS) and DeltaGraph Pro $^{\oplus}$ software programs.

\section{RESULTS}

\section{Behavioral Effects of AMPT}

AMPT, in comparison to diphenhydramine, did not produce an exacerbation of depressed mood in the patients. The ANOVA of mean HDRS scores revealed a nonsignificant main effect of $\operatorname{drug}(F=1.2, d f 1, p=.285)$, but a significant main effect of time ( $F=4.39, d f 3, p=$ $.008)$, and a significant interaction of drug and time ( $F=$ $3.74, d f 3, p=.017)$. Post hoc analysis indicated a statistically significant decrease in HDRS score during diphenhydramine administration on day 3 , and on the posttest evaluation (day 4), compared to baseline. The change in HDRS was modest, however, and not clinically meaningful. There were no significant changes in HDRS at any time after AMPT administration (see Figure 1). The items on the HDRS that had significant treatment $\times$ time interaction were psychic anxiety, loss of energy, loss of interest, and pathologic guilt. These items decreased during control testing. One item of the HDRS, early morning awakening, was significantly less during AMPT testing than control. It is noteworthy that none of the 17 patients experienced clinically meaningful changes in mood during or after AMPT administration.

The only significant changes identified by ANOVA on the visual analogue scales were for the mood states "tired" and "energetic." AMPT, in comparison to diphenhydramine, made the patients feel less energetic $(F=$ $2.9, d f=3, p<.05)$ and more tired $(F=2.8, d f=3, p<.05)$.

\section{Effect of AMPT on Plasma MHPG and HVA Levels}

As expected, AMPT produced robust and significant decreases in plasma MHPG and HVA. The ANOVA for plasma MHPG revealed significant main effects of drug $(F=27.8, d f=1, p<.001)$, time $(F=17.9, d f=3, p<$ $.001)$, and drug and time interaction $(F=13.0, d f=3, p<$ .001). Plasma MHPG was significantly decreased on day 3 and 4 (Figure 2). Similarly, the ANOVA for plasma HVA revealed significant effects of drug $(F=6.3, d f=1$, $p<.03)$, time $(F=12.7, d f=3, p<.001)$, and the drug and time interaction $(F=18.9, d f=3, p<.001)$. Plasma HVA was significantly decreased on day 3 and day 4 (Figure 3). The maximum decrease in plasma HVA was $10 \mathrm{mg} / \mathrm{ml}$ (day 3 minus day 1 ) and the maximum 


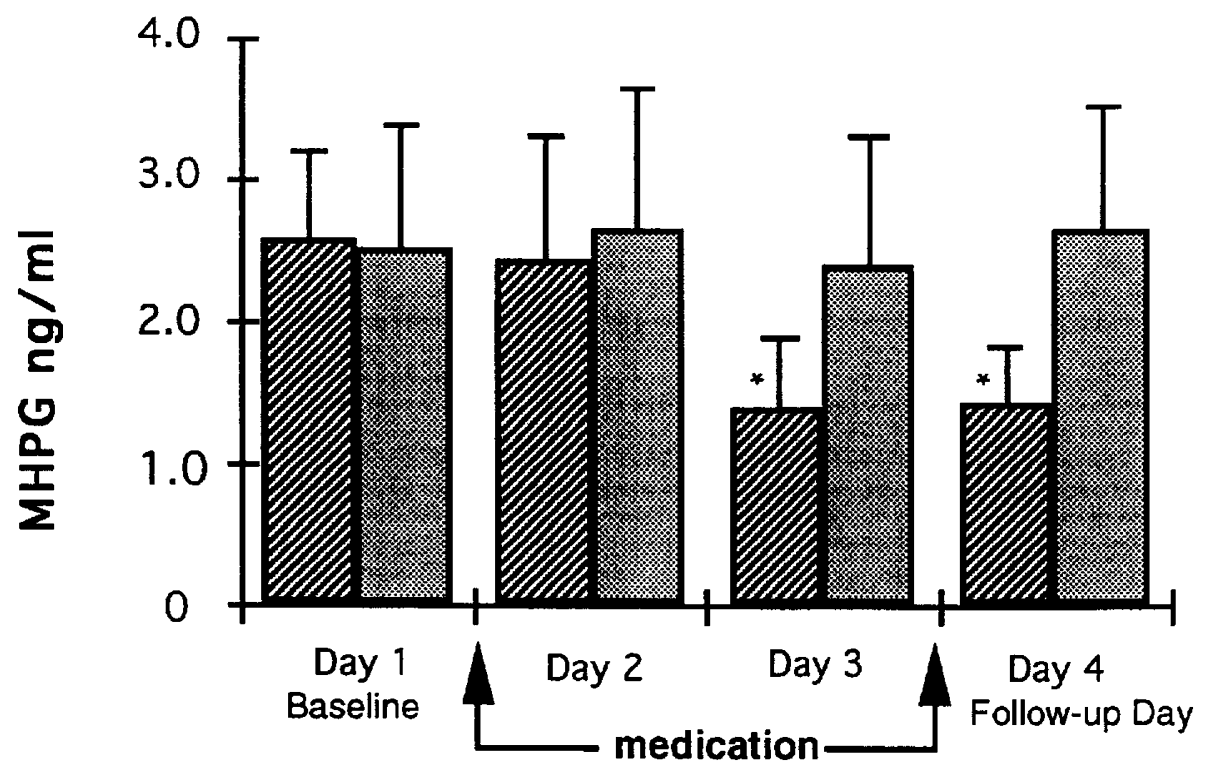

AMPT or Diphenhydramine Administration
Figure 2. The effect of AMPT (striped bars) and diphenhydramine (shaded bars) on plasma MHPG levels. There was a significant drug and time interaction ( $F$ $=13.0, d f=3, p<.001)$. There were significant decreases $(p<.01)$ in plasma MHPG levels during AMPT administration, as indicated by the asterisks. There were no significant decreases in plasma MHPG during diphenhydramine administration. decrease in plasma MHPG was $1.3 \mathrm{mg} / \mathrm{ml}$ (day 3 minus day 1 ), a $10 \%$ and $50 \%$ reduction, respectively.

\section{DISCUSSION}

These findings suggest that acute reduction of brain concentrations of NE and DA do not increase the symptoms of depression in drug-free symptomatic depressed patients. The validity of this interpretation depends upon the ability of AMPT to lower brain levels of NE and DA. Preclinical studies have demonstrated that AMPT produces a reduction in brain biosynthesis and concentrations of catecholamines (Widerlov and Lewan- der 1978). The observation that AMPT in the present study decreased plasma MHPG and HVA levels and in previous clinical studies reduced urinary (Brodie et al. 1971; Engelman et al. 1968; Sjoerdsma et al. 1965) and cerebrospinal fluid catecholamine metabolites (Brodie et al. 1971) is consistent with the preclinical findings of reduced catecholamine concentrations after AMPT administration. Brodie reported that AMPT doses greater than $2 \mathrm{~g} /$ day decreased urinary vanillylmandelic acid (VMA) by $25 \%$ and MHPG by $33 \%$. AMPT doses of $4 \mathrm{~g} /$ day decreased spinal fluid HVA by more than $40 \%$ (Brodie et al. 1971). Engelman reported that AMPT doses greater than $1 \mathrm{~g} /$ day reduced urinary VMA by $50 \%$ (Engelman et al. 1968).

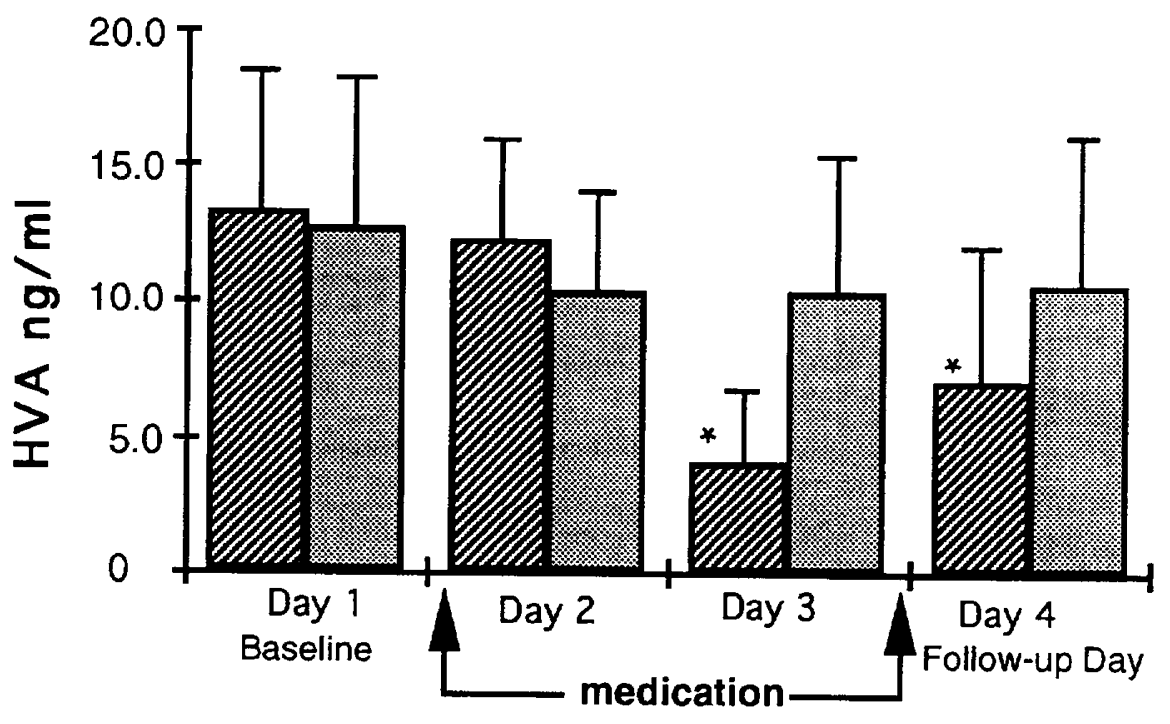

AMPT or Diphenhydramine Administration
Figure 3. The effect of AMPT (striped bars) and diphenhydramine (shaded bars) on plasma HVA levels. There was a significant drug and time interaction $(F=$ $18.9, d f=3, p<.001)$. There were significant decreases $(p$ $<.01$ ) in plasma HVA levels during AMPT administration, as indicated by the asterisks. There were no significant decreases in plasma HVA during diphenhydramine administration. 
It is important to emphasize that the current study investigates the effects of a short-term reduction of NE and DA synthesis. It does not address the consequences of long-term synthesis inhibition or alterations in monoamine storage, release, or monoamine receptor activity.

AMPT has not been reported to produce major alterations in mood in medical patients (Engelman et al. 1968; Sjoerdsma et al. 1965). To our knowledge, the only previous study of the behavioral effects of AMPT in drug-free depressed patients are the reports of Bunney and colleagues (Brodie et al. 1971). They found that AMPT worsened the symptoms of three out of four psychotically depressed patients. This exacerbation was reversed by discontinuation of the AMPT. The discrepancy between this study and the present one may be due to patient characteristics. For example, none of our patients were psychotic. Alternatively, as noted by others (Mendels and Frazer 1974), these reported effects of AMPT may simply have been a sedative effect rather than a primary action on depressed mood.

A major premise of the original catecholamine deficiency hypothesis of depression was the reported moodlowering effects of catecholamine depleting antihypertensive drugs, such as reserpine. However, subsequent reviews have concluded that reserpine produces symptoms of depression in only a small percentage of patients with a history of affective illness (Bunney et al. 1971; Mendels and Frazer 1974).

The data regarding a possible association between depression and the use of $\beta$-adrenergic receptor antagonist drugs are conflicting (Avorn et al. 1986; Bright and Everitt 1992; Carney et al. 1987; Dimsdale et al. 1989; Paykel et al. 1982; Thiesen et al. 1990). There is little evidence from prospective clinical trials to causally link $\beta$-receptor antagonist administration and the development of clinical depression. Further, studies designed to test the therapeutic significance of the catecholamine and indoleamine deficiency hypotheses by evaluating the effectiveness of catecholamine and indoleamine precursors in treatment of depression have generally been negative, with L-DOPA and L-tryptophan exhibiting little efficacy in depressed patients (Charney and Delgado 1992; Goodwin et al. 1970).

One explanation for the lack of effect of AMPT on depressive symptoms was that the patients were already severely depressed and there was a ceiling effect. This is possible, although patients with HDRS scores in the low to mid 20's did not experience an exacerbation of depression.

The results from this and other studies provide an opportunity to critically reexamine the role of brain monoamine function in the pathophysiology of depression and the mechanism of antidepressant drug action. Previous investigations have found that depletion of tryptophan, with resultant lowering of brain serotonin levels, does not produce marked alterations of mood in drug-free depressed patients (Delgado et al. 1994). Further, we have preliminary data indicating that combined administration of AMPT (depleting NE and DA) and a tryptophan-depleting amino acid drink (lowering serotonin) does not produce depressive symptoms in eight healthy subjects (RMS, HLM, DSC unpublished observations, May 1994). However, both tryptophan depletion and AMPT have been shown to reverse the therapeutic effects of selective serotonin reuptake inhibitors and selective NE reuptake inhibitors, respectively (Delgato et al. 1990; Miller et al. in press). Thus, alterations in serotonin, DA, and NE systems may be relevant to the mechanisms of antidepressant action rather than reflective of the primary pathophysiology of depressive illness. One alternative explanation is that in drug-free depressed patients these neurotransmitter systems are maximally decreased, such that further manipulations do not worsen depressive symptoms. This explanation may not be correct because of the clinical observation that catecholamine precursors are ineffective treatments for depression. Given that currently available antidepressant drugs have major effects on the brain's monoamine systems, the primary pathology of depression may be due to an abnormality in another neuronal system, which remains to be identified, that is highly regulated by these brain systems.

\section{ACKNOWLEDGMENTS}

This study was funded by grants from the Department of Veterans Affairs (HLM, PLD, DSC). HVA and MHPG assays were performed by Harold Landis and Robert Reynolds at the Yale Mental Health Clinical Research Center, Gas Chromatography-Mass Spectroscopy Laboratory. The assistance of Lisa Roach, Kathleen Colonese, and Melissa Giunti in conducting this study is gratefully acknowledged.

\section{REFERENCES}

Avorn J, Everitt DE, Weiss S (1986): Increased antidepressant use in patients prescribed $\beta$-blockers. JAMA 255:357-360

Bacopoulos NG, Redmond DE, Roth RH (1979): Serotonin and dopamine metabolites in brain regions and cerebrospinal fluid of a primate species: Effects of ketamine and fluphenazine. J Neurochem 32:1215-1218

Bright RA, Everitt DE (1992): $\beta$-blockers and depression: Evidence against an association. JAMA 267:1783-1787

Brodie HKH, Murphy DL, Goodwin FK, Bunney WE Jr (1971): Catecholamines and mania: The effect of $\alpha$-methylpara-tyrosine on manic behavior and catecholamine metabolism. Clin Pharmacol Ther 112(2):218-224

Bunney WE Jr, Brodie KH, Murphy DL, Goodwin FK (1971): Studies of $\alpha$-methyl-para-tyrosine, L-DOPA, and L-TRYPTOPHAN in depression and mania. Am J Psychiatry 127(7):872-881

Bunney WE Jr, Gershon ES, Murphy DL, Goodwin FK (1972): 
Psychobiologic and pharmacologic studies of manicdepressive illness. J Psychiatry Res 9:207-226

Bunney WE, Davis JM (1966): Norepinephrine in depressive reactions: A review. Arch Gen Psychiatry 13:483-494

Carney RM, Rich MW, Tevelde A, Saini J, Clark K, Freedland KE (1987): Prevalence of major depressive disorder in patients receiving beta blocker therapy versus other medications. Am J Med 83:223-226

Charney DS, Delgado PL (1992): Current concepts of serotonin neuronal function and the pathophysiology of depression. In Elliott M, Heal DJ, Marsden CA (eds), Experimental Approaches to Anxiety and Depression. London, John Wiley \& Sons, Ltd

Delgado PL, Charney DS, Price LH, Aghajanian GK, Landis H, Heninger GR (1990): Serotonin function and the mechanism of antidepressant action: Reversal of antidepressant induced remission by rapid depletion of plasma tryptophan. Arch Gen Psychiatry 47:411-418

Delgado PL, Price LH, Miller HL, Salomon RM, Aghajanian GK, Heninger GR, Charney DS (1994): Serotonin and the neurobiology of depression: Effects of tryptophan depletion in drug-free depressed patients. Arch Gen Psychiatry 51:865-874

Delgado PL, Price LH, Heninger GR, Charney DS (1992): Neurochemistry of affective disorders. In Paykel ES (ed), Handbook of Affective Disorders. New York, Churchill Livingstone

Dimsdale JE, Newton RP, Joist T (1989): Neuropsychological side effects of beta blockers. Arch Intern Med 149:514-525

Engelman K, Horwitz D, Jequier E (1968): Biochemical and pharmacological effects of $\alpha$-methyltyrosine in man. J Clin Invest 47:577-594

Garcia-Sevilla JA, Guimon P, Garcia-Vallejo P, Fuster MJ (1966): Biochemical and functional evidence of supersensitive platelet $\alpha$-2-adrenoreceptors in major affective disorder: Effect of long-term lithium carbonate treatment. Arch Gen Psychiatry 43:51-57

Goodwin FK, Murphy DL, Brodie KH, Bunney WE Jr (1970): L-DOPA, catecholamines, and behavior: A clinical and biochemical study in depressed patients. Biol Psychiatry 2:341-366

Kapur S, Mann JJ (1992): Role of the dopaminergic system in depression. Biol Psychiatry 32:1-17
Maas JW, Hattox SE, Landis DH, Roth RH (1976): The determination of a brain arteriovenous difference for 3-methoxy-4-hydroxyphenylethylene glycol (MHPG). Brain Res 118:167-173

Mann JJ, Stanley M, McBride PA, McEwen BS (1986): Increased serotonin 2 and $\beta$-adrenergic receptor binding in the frontal cortices of suicide victims. Arch Gen Psychiatry 43:954-959

Mazure C, Nelson C, Price LH (1986): Reliability and validity of the symptoms of major depressive illness. Arch Gen Psychiatry 43:451-456

Mendels J, Frazer A (1974): Brain biogenic amine depletion and mood. Arch Gen Psychiatry 30:447-451

Miller HL, Delgado PL, Salomon RM, Berman R, Krystal JH, Heninger GR, Charney DS (1995): The behavioral effects of catecholamine depletion on antidepressant induced remission of depression: Implications for monoamine hypothesis of antidepressant action. Arch Gen Psychiatry (in press)

Paykel ES, Fleminger R, Watson JP (1982): Psychiatric side effects of antihypertensive drugs other than reserpine. $J$ Clin Psychopharmacol 2:14-39

Schildkraut JJ (1965): The catecholamine hypothesis of affective disorders: A review of supporting evidence. Am J Psychiatry 122:509-522

Siever LJ (1987): Role of noradrenergic mechanisms in the etiology of the affective disorders. In Meltzer HY (ed), Psychopharmacology: The Third Generation of Progress. New York, Raven

Siever LJ, Davis KL (1985): Overview: Toward a dysregulation hypothesis of depression. Am J Psychiatry 142:1017-1031

Sjoerdsma A, Engelman K, Spector S (1965): Inhibition of catecholamine synthesis in man with $\alpha$-methyl-tyrosine, an inhibitor of tyrosine hydroxylase. Lancet 2:1092-1094

Thiesen BQ, Wallace SM, Blackburn JL, Wilson TW, Bergman UIF (1990): Increased prescribing of antidepressants subsequent to $\beta$-blocker therapy. Arch Intern Med 150: 2286-2290

Widerlov E, Lewander T (1978): Inhibition of the in vivo biosynthesis and changes of catecholamine levels in rat brain after $\alpha$-methyl-p-tyrosine; time- and dose-response relationships. Naunyn-Schmiedeberg's Arch Pharmacol 304:111-123 